\title{
Design of new ultrasonic transducer with two elements for flow rate measurement using ultrasonic Doppler method
}

\author{
Jevin Tanius Owen ${ }^{1, *}$, Ari Hamdani ${ }^{2}$, Tomonori Ihara $^{3}$, Hideharu Takahashi $^{2}$, and Hiroshige Kikura ${ }^{2}$ \\ ${ }^{1}$ Department of Mechanical Engineering, Tokyo Institute of Technology, 2-12-1, Ookayama, Meguro-ku, Tokyo, Japan \\ ${ }^{2}$ Laboratory for Advanced Nuclear Energy, Tokyo Institute of Technology, 2-12-1, Ookayama, Meguro-ku, Tokyo, Japan \\ ${ }^{3}$ Tokyo University of Marine Science and Technology, 2-1-6 Etchujima, Koto-ku, Tokyo, Japan
}

\begin{abstract}
In this present study, we developed a new two-dimensional velocity profile measurement system by utilizing two-elements ultrasonic transducer with both elements acting as transmitter and receiver. The aim is to obtain two-dimensional velocity information along measurement line and calculate the flow rate. We evaluate the transducer with sound pressure measurement and flow measurement. From the sound pressure measurement, there is one main lobe exist at the center between the elements due to the interference of both waves generated from each element. Therefore two-dimensional velocity measurement is possible. From the flow measurement, we can get two-dimensional velocity profile and calculate the flow rate. We compared the flow rate result with the flow rate reading from the electromagnetic flow meter, and we obtained the error of $4.98 \%$ for pipe flow and $10.43 \%$ for pipe flow under swirl effect with the measurement range from $8 \mathrm{~mm}$ to $50 \mathrm{~mm}$. We used B-spline interpolation with Gaussian fitting to estimate the profile from range $0 \mathrm{~mm}$ to $8 \mathrm{~mm}$ and finally we were able to obtain full vector profile.
\end{abstract}

\section{Introduction}

Ultrasonic Doppler method was first used in medical field. Applying this method, Baker [1] performed the blood flow sensing. This application utilized a onedimensional technique which has some limitations. For instance, in blood flow sensing, the "real" velocity is extracted from the velocity in ultrasound beam direction by assuming the blood flow parallel to vessel axis. In many areas of clinical interest, however, complex flow patterns, such as helices, shear layers, recirculation zones, and turbulence are present, making assumptions about the flow direction difficult and suspect [2]. Therefore, multi-dimensional velocity measurement is necessary. Peronneau et al. [3] proposed single element cross beam system using two transducers as a transceiver (transmitter/receiver). However, this system can only measure two-dimensional velocity at one point, and mechanical adjustment is needed to measure another point. Later, Scabia et al. [4] proposed multiple elements cross beam system by using 128 elements array transducer with at least one transmitter and two receivers to measure at one point.

In fluid engineering, Takeda [5] was first to apply ultrasonic Doppler method for measuring onedimensional velocity profile in pipe flow. This technique then has a recognition as a powerful tool in fluid engineering for its ability of non-intrusiveness and broad range application, including opaque liquid. Wada et al. [6] applied this method for flow rate measurement in power plant using multi-lines measurement with cavitation bubbles system and metallic pipe wall. In this field, however, complex flow (e.g., non-axisymmetric flow) exists too. Due to this concern, Hurther and Lemmin [7] developed three-dimensional velocity measurement in open-channel flow using one transmitter and four receivers. Based on this idea, Obayashi et al.[8] developed two-dimensional measurement system using only one transceiver and one receiver. They investigated the system accuracy using a rotating cylinder with known rotation speed and distance of measurement line from center. They can measure two-dimensional velocity in a certain depth, but they found the velocity in receiver measurement line with the high error in limited depth. The reason is the echo obtained in the receiver is side scattering echo from transmitter measurement line which has low signal to noise ratio unlike the back scattering echo in transmitter line.

Through the previous cases learning, this present study proposes new measurement system with two elements array transducer to measure two-dimensional velocity profile. Each element acts as a transceiver so that we expect no side scattering occurred. Instead of using crossbeam system, we pursue an interference of both waves generated from each element to produce one main lobe or one measurement volume at the center

\footnotetext{
Corresponding author: tanius.j.aa@m.titech.ac.jp
} 
between the elements. With this measurement system configuration, the required number of the pulse generators is only two. Therefore it can reduce the measurement equipment size and electronic cost.

\section{Measurement principle}

\subsection{Single element transducer velocity profiler}

In this study, the ultrasonic flow monitor employs ultrasonic Doppler method to provide spatial-temporal velocity information. The ultrasonic transducer transmits a mechanical wave repeatedly, and if a reflector (e.g., bubble or particle) exists along the wave propagation, there will be echoes repeatedly reflected back to the element (see Figure 1). This echo contains information about particle position $(x)$ by the delayed time $(T)$ between wave transmission and reception, while the consecutive echo contains information about reflector velocity (fluid velocity, if their density same) in the wave propagation direction $\left(V_{w}\right)$ by the Doppler frequency $\left(f_{d}\right)$ estimation [9] with equation below.

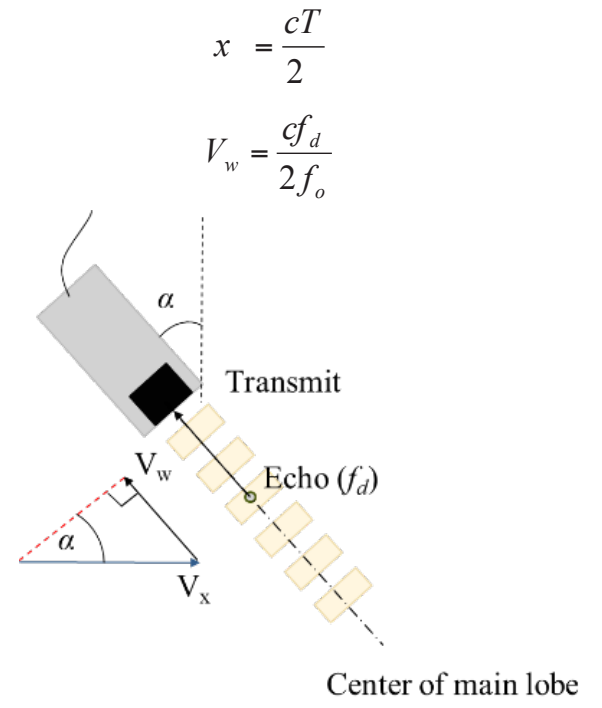

(a) One-dimensional velocity measurement.

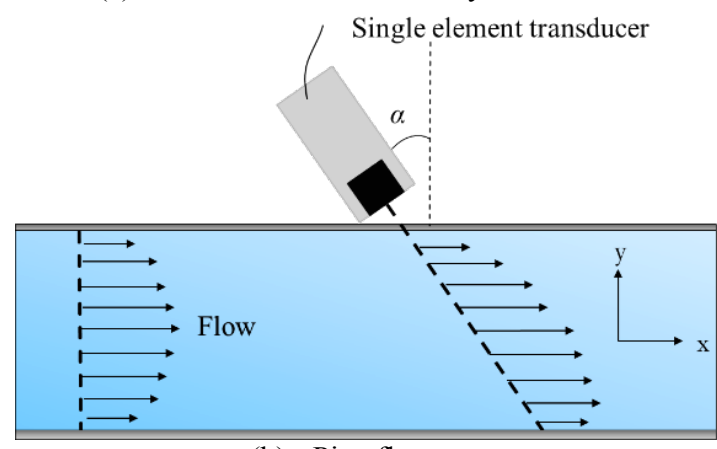

(b) Pipe flow measurement.

Fig.1. Single element transducer profiler.

The variable $c$ and $f_{o}$ represent sound velocity (e.g., $1480 \mathrm{~mm} / \mathrm{s}$ in water at $20^{\circ} \mathrm{C}$ ) and transducer basic frequency respectively. In the single element transducer measurement, the transducer is set up with the particular incident angle $(\alpha)$. The velocity of the fluid $\left(V_{x}\right)$ can be calculated by the projection of the velocity in the wave propagation direction $\left(V_{w}\right)$ to the fluid velocity by assuming that the flow is one-dimensional and parallel to the pipe axis. Another assumption is the difference of the velocity profile in the measurement line, and the velocity profile in the flow at one position (straight line) is negligible (suitable in fully developed flow). Finally, the flow profile can be obtained, and the flow rate can be calculated by the integration of the velocity profile of the fluid in the pipe with a certain radius $(R)$. The fluid velocity and flow rate are represented by the equation below.

$$
\begin{gathered}
V_{x}=\frac{V_{w}}{\sin \alpha} \\
Q=\pi \int_{-R}^{R} V_{x} r d r
\end{gathered}
$$

\subsection{Two elements transducer velocity profiler}

The mechanism of the measurement using two elements transducer is similar to single element transducer, except the echo is received by both elements. Hence there is two Doppler frequency with a different orientation (see Figure 2), and two-dimensional velocity can be reconstructed as below.

$$
\begin{gathered}
V_{x}=\frac{c}{2 f_{o}} \frac{f_{d 1}-f_{d 2}}{\sin \theta} \\
V_{y}=\frac{c}{2 f_{o}} \frac{f_{d 1}+f_{d 2}}{1+\cos \theta}
\end{gathered}
$$

Using this principle, we can obtain two-dimensional velocity profile without adopting the assumption in single element transducer measurement. In fact, the velocity magnitude $(V)$ and angle $(\beta)$ of the flow can be calculated with the equation below.

$$
\begin{aligned}
& V=\sqrt{V_{x}^{2}+V_{y}^{2}} \\
& \beta=\tan ^{-1}\left(\frac{V_{y}}{V_{x}}\right)
\end{aligned}
$$

Another important thing to be noted, that this measurement occurs in 1 position (the measurement line is perpendicular to the pipe axis), unlike single element transducer. Therefore, when the flow is not fully developed (e.g., transient flow in short entry length or swirling flow), the flow rate (using equation (4)) is better calculated from velocity profile at one particular position.

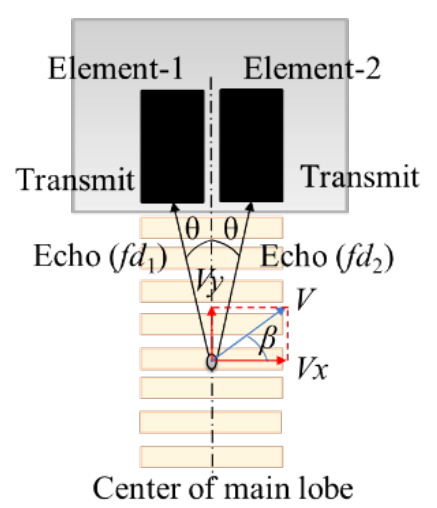

(a) Two-dimensional velocity measurement. 


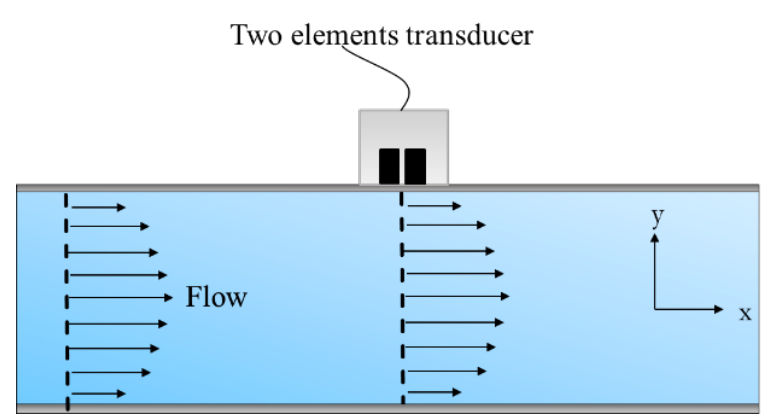

(b) Pipe flow measurement.

Fig.2. Two elements transducer profiler.

\section{Design of two elements transducer}

\subsection{Design consideration}

The primary objective of the design is to measure twodimensional velocity profile. However, it does not simply succeed by using two transducers or two elements array transducer transmitting together. For example, if we use two transducers (i.e., $4 \mathrm{MHz}$, diameter $5 \mathrm{~mm}$ ) attached to each other and transmitting together, there are two existing main lobe or measurement volume, and two-dimensional velocity measurement is not possible (see Figure 3).

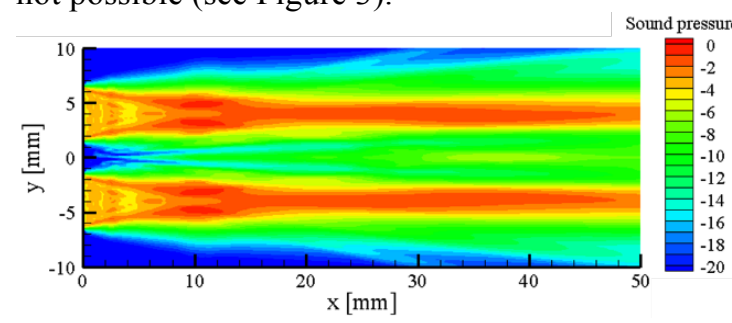

Fig.3. Sound pressure simulation of two transducers.

To ensure the quality of the measurement, we set the design target which is one main lobe in a good intensity and directivity with the acceptable near field length. To achieve this target, we do some parametric study with the parameters that have a significant contribution, i.e., basic frequency of transducers $\left(f_{o}\right)$, element width $(a)$ and inter-element spacing $(d)$. Figure 4 illustrates the transducer design parameters

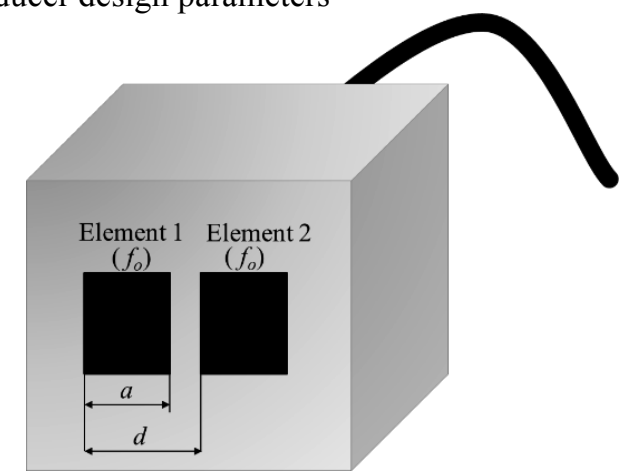

Fig.4. Design parameters of two elements transducer.

This parameters selection will decide the measurement quality or to achieve design targets. To get one main lobe in measurement, we have to choose a small inter-element spacing $(d)$ and consequently a small element width $(a)$ too with sufficient intensity as a constraint. To obtain a good intensity and directivity, we have to choose the high basic frequency (the option is $2 \mathrm{MHz}, 4 \mathrm{MHz}$, and $8 \mathrm{MHz}$ ). However, the high basic frequency will result in a long near field zone which is not suitable for measurement. Therefore, we need to compromise the design parameters and select the best configuration for optimum measurement.

\subsection{Design selection and simulation}

Through the parametric study and sound pressure simulation using software based on Matlab code, we finally decided the design parameters for the transducer as in Table. 1 below.

Table 1. Transducer design specification.

\begin{tabular}{|c|c|}
\hline Transducer detail & Specification \\
\hline Basic frequency $\left(f_{o}\right)$ & $4 \mathrm{MHz}$ \\
\hline Number of element & 2 \\
\hline Element width $(a)$ & $2.5 \mathrm{~mm}$ \\
\hline Inter-element spacing $(d)$ & $2.7 \mathrm{~mm}$ \\
\hline
\end{tabular}

For basic frequency, we had to compromise intensity and directivity with near field length. Therefore $4 \mathrm{MHz}$ was chosen to attain a good intensity and directivity while having an acceptable near field length. As for the element width and inter-element spacing, we used the value in Table 1 and tried to simulate the sound pressure. The result is the transducer generating one main lobe in the center between both elements, In addition, a good comparison to commercial single element transducers design (4 MHz, $5 \mathrm{~mm}$ ) is shown (see Figure 5). Based on the simulation data, two-dimensional velocity profile measurement is possible to be carried out. As good as the simulation data, however, we need to confirm it by the experiment of the sound pressure and flow measurement. Finally, we got the design transducer to be manufactured (see Figure 6) by Japan Probe Co, Ltd. and evaluated the design by experiment.

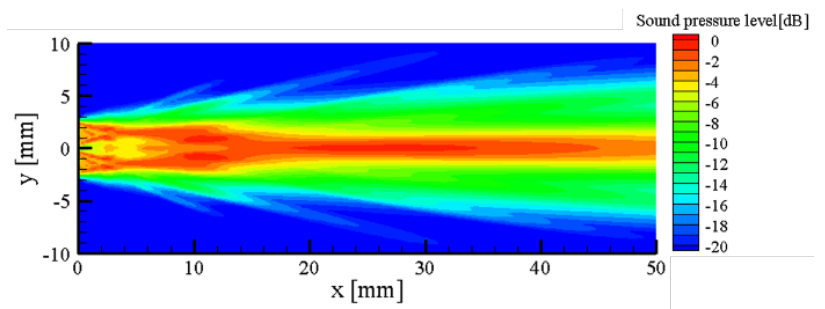

(a) Two-elements transducer $4 \mathrm{MHz}$

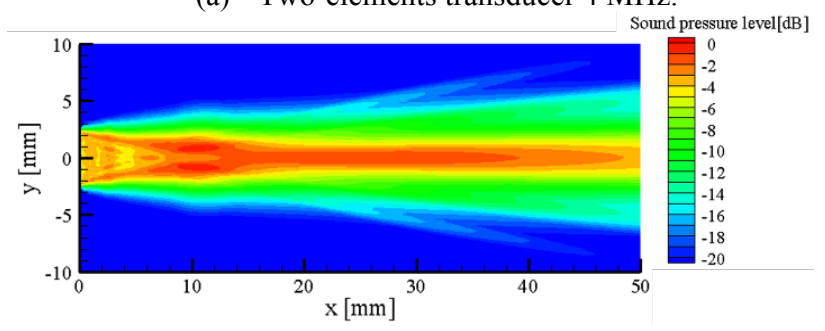

(b) Single-elements transducer $4 \mathrm{MHz}$.

Fig.5. Sound pressure simulation data. 


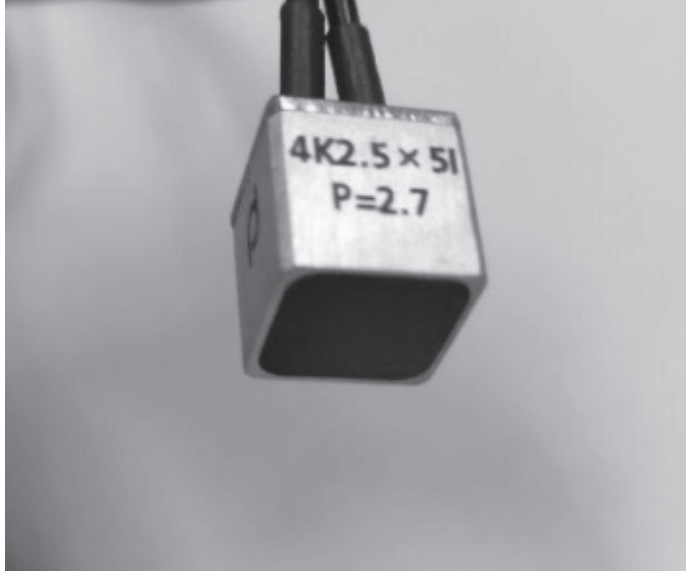

Fig.6. Two elements transducer.

\section{Sound pressure measurement}

\subsection{Experimental setup and procedures}

To confirm the simulation data, sound pressure measurement was conducted in the water tank using two elements transducer transmitting while needle hydrophone is receiving. Figure 7 shows the experimental setup. The transducer is controlled by pulse generator two channel (Japan Probe TIT-10B-USB). The needle hydrophone is mounted at $\mathrm{XYZ}$ stage controller which connected to $\mathrm{PC}$ with $\mathrm{A} / \mathrm{D}$ converter. Using this system, the needle hydrophone can be moved, and the sound pressure measurement can be done in particular grid and position (two-dimensional) by controlling the stage controller with the condition in Table 2.

Table 2. Sound pressure measurement condition.

\begin{tabular}{|c|c|}
\hline Condition & Detail \\
\hline Water temperature & $23.7^{\circ} \mathrm{C}$ \\
\hline Sampling profile & 50 \\
\hline Axial distance $(x)$ & $50 \mathrm{~mm}$ \\
\hline Half lateral distance $(y)$ & $10 \mathrm{~mm}$ \\
\hline Axial resolution $(\Delta x)$ & $1 \mathrm{~mm}$ \\
\hline Lateral resolution $(\Delta y)$ & $0.5 \mathrm{~mm}$ \\
\hline
\end{tabular}

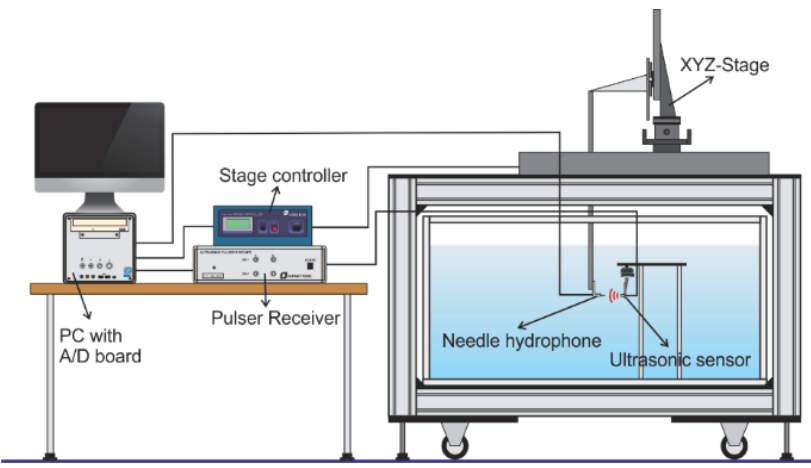

(a) Apparatus setup.

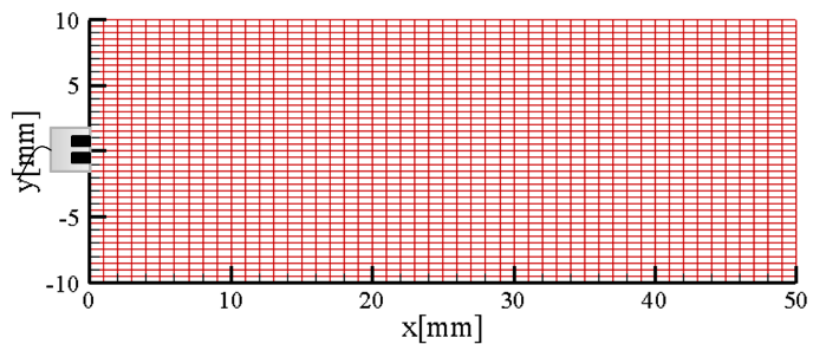

(b) Measurement grid (resolution).

Fig.7. Sound pressure measurement by experiment.

The signal transmitted from the transducer is recorded by needle hydrophone in sound intensity $(I)$, peak to peak voltage. Then the data is plotted on a color graph by using the Tecplot software after converted into sound pressure level $(S P L)$ using the equation below.

$$
S P L=20 \log _{10} \frac{I}{I_{\max }}[d B]
$$

\subsection{Results and discussion}

The sound pressure measurement is being done by using only one element at first to check each element, and then two elements transmitting together. The result is plotted on a color graph and compared to simulation data as below.

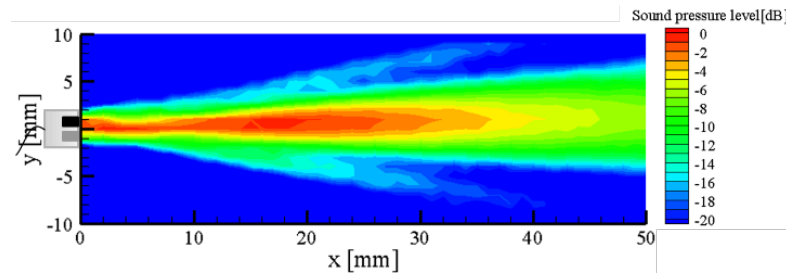

(a) Element-1.

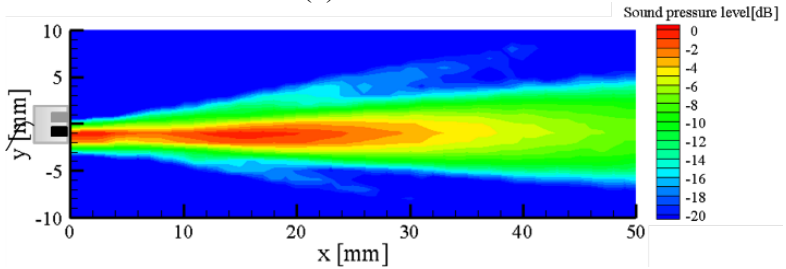

(b) Element-2.

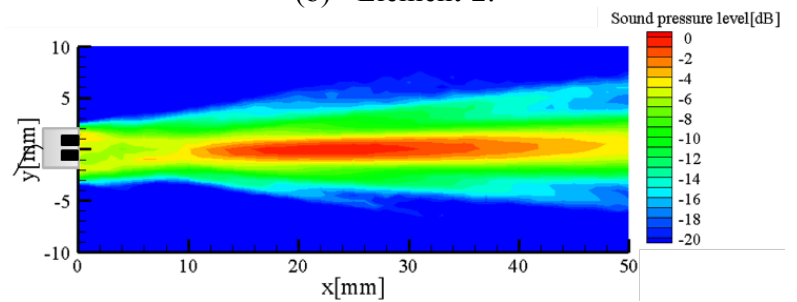

(c) Two elements.

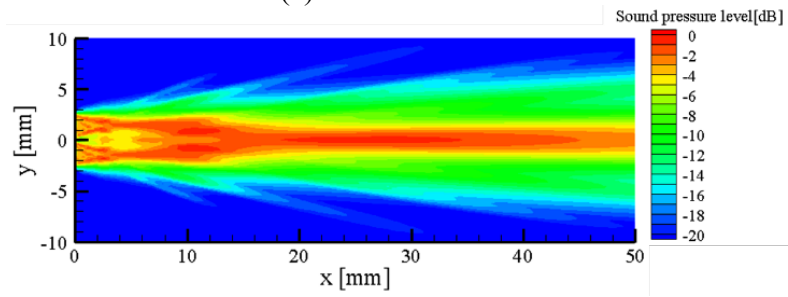

(d) Simulation of two elements.

Fig.8. Sound pressure measurement of two element transducer. 
From the result of one element transmitting, we confirm the both elements are working fine. It can be noticed that from one element transmitting, the main lobe is in the center of each element. When the two elements are transmitting together, there is interference of both waves from each element which resulting one main lobe in the center between of the both elements. However, if we notice from the sound pressure of two elements transmitting together in the axial distance $(x)$ up to around $8 \mathrm{~mm}$, the sound pressure shows a lower intensity in the center compared to upper and lower lateral distance $(y)$. In this region, it is suspected that the measurement volume is not one focused in the center. For example, the data is extracted at axial distance $(x)$ of $5 \mathrm{~mm}$ and $15 \mathrm{~mm}$ (see Figure 9). In the data from the axial distance of $5 \mathrm{~mm}$, we can see that there are two peaks of intensity which indicates the measurement volume is not one focused in the center. On the contrary, the data from the axial distance of $15 \mathrm{~mm}$ shows one peak in the center (one measurement volume). Therefore two-dimensional velocity measurement might not be effective in the region with two peaks of intensity (up to $8 \mathrm{~mm}$ ). There is a difference from the sound pressure data of the experiment and simulation, if we observe the intensity in the center $(y=0)$ along the axial distance (see Figure 10). For example, from the simulation data we can observe the near field effect from the oscillating intensity. This difference might be caused by the parameters (i.e., resolution, number of sample, sampling method, attenuation and etc.) between simulation and experiment are not the same. Nevertheless, with the sound pressure data from the experiment, we confirm our design target fulfilled as expected in the simulation data and the design could attain two-dimensional velocity in the flow measurement.

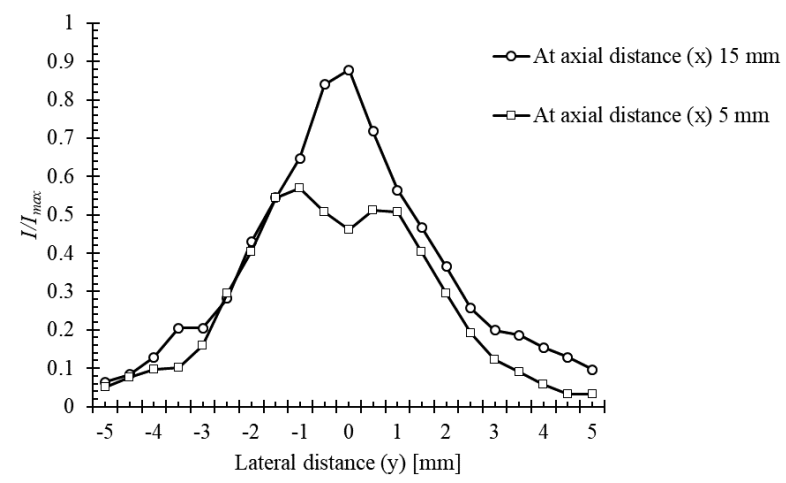

Fig.9. Normalized sound intensity along lateral distance (y).

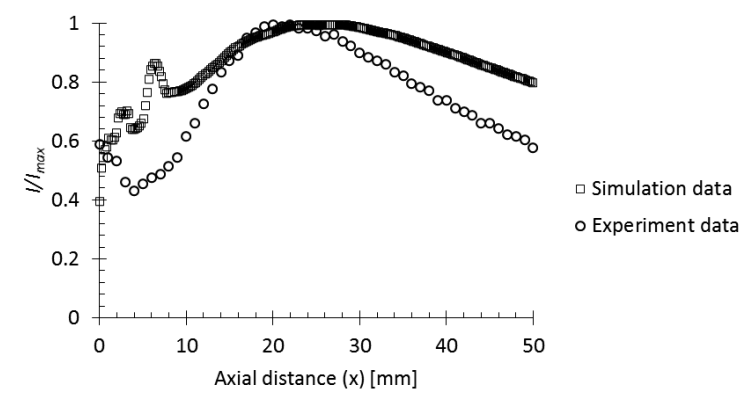

Fig.10. Normalized sound intensity in the center $(y=0)$.

\section{Flow measurement}

\subsection{Experimental setup and procedures}

The flow measurement was conducted to confirm the capability of measuring the two-dimensional velocity. The experiment was being carried out in the horizontal acrylic pipe with and without swirling effect using swirl generator (rotating pipe driven by motor). The working fluid is tap water with the temperature controlled by the cooling system (see Figure 11). We used nylon particle (diameter $80 \mu \mathrm{m}$ ) as a reflector which has a similar density as water $\left(1.03 \mathrm{~g} / \mathrm{cm}^{3}\right)$. Therefore it is expected to have the same velocity with water. For the flow rate reading, we used electromagnetic flow meter as the reference and controlled by the ball valve. The transducer is placed in the test section with the pipe thickness of $1 \mathrm{~mm}$ and immersed in water as coupling fluid to minimize the attenuation of wave propagation to the working fluid. We used two channel pulse generator (Japan Probe TIT-10B-USB), A/D converter (National Instrument PXI-1033) connected to PC and controlled by developed software. The experiment condition and measurement parameters are presented in Table 3 and Table 4. The measurement data is then plotted as velocity profile (axial and radial) and velocity vector profile. For this present study, we compared only axial velocity for flow rate calculation. Therefore we used single element transducer to get the axial velocity to compare the profile. After that, we calculated the flow rate from each profile and compared them to the flow rate reading of the electromagnetic flow meter.

Table 3. Flow measurement condition.

\begin{tabular}{|c|c|}
\hline Condition & Detail \\
\hline Water temperature & $28 \pm 1^{\circ} \mathrm{C}$ \\
\hline Flow rate (reading) & $35 \mathrm{~L} / \mathrm{min}$ \\
\hline Reynold number & 18000 \\
\hline Pipe inner diameter & $50 \mathrm{~mm}$ \\
\hline Motor frequency (in swirling case) & $11.9 \mathrm{~Hz}$ \\
\hline Test section from motor & $3 \mathrm{D}$ \\
\hline Test section pipe material & Acrylic \\
\hline
\end{tabular}

Table 4. Measurement parameters.

\begin{tabular}{|c|c|}
\hline Parameter & Detail \\
\hline Basic frequency & $4 \mathrm{MHz}$ \\
\hline Pulse repetition frequency & $1 \mathrm{kHz}$ \\
\hline Channel width & $0.74 \mathrm{~mm}$ \\
\hline Channel distance & $0.74 \mathrm{~mm}$ \\
\hline Number of cycles & 4 \\
\hline Number of repetitions & 128 \\
\hline Number of profile & 5000 \\
\hline Voltage & $120 \mathrm{~V}$ \\
\hline Gain & $30 \mathrm{~dB}$ \\
\hline
\end{tabular}




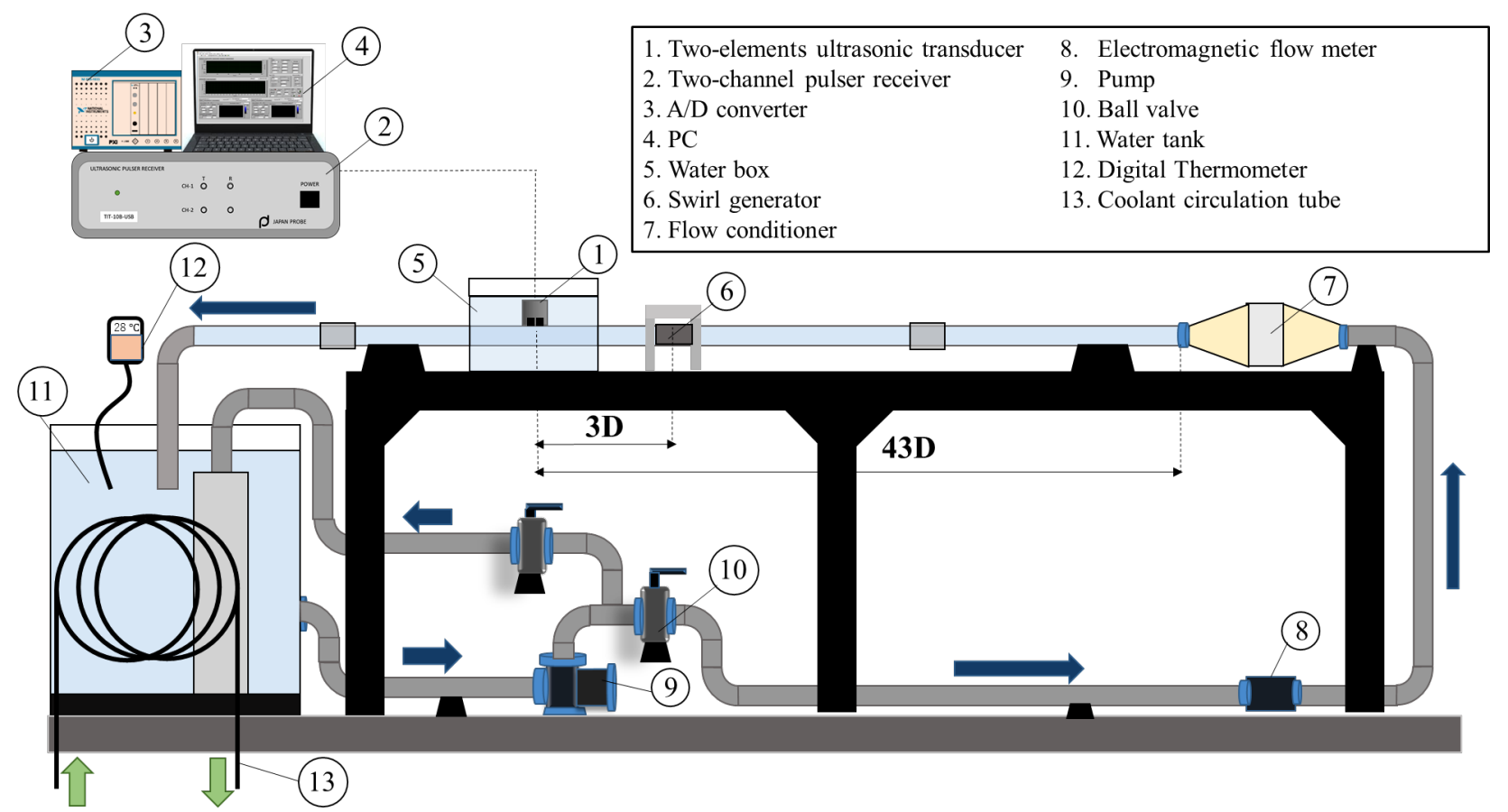

Fig.11. Flow measurement apparatus.

\subsection{Results and discussion}

\subsubsection{Pipe flow without swirl effect}

The two-dimensional velocity in the turbulent pipe flow without swirl effect was measured. The result is presented in time averaged velocity profile graph (5000 profiles) with the standard deviation and reconstructed into vector velocity as below.

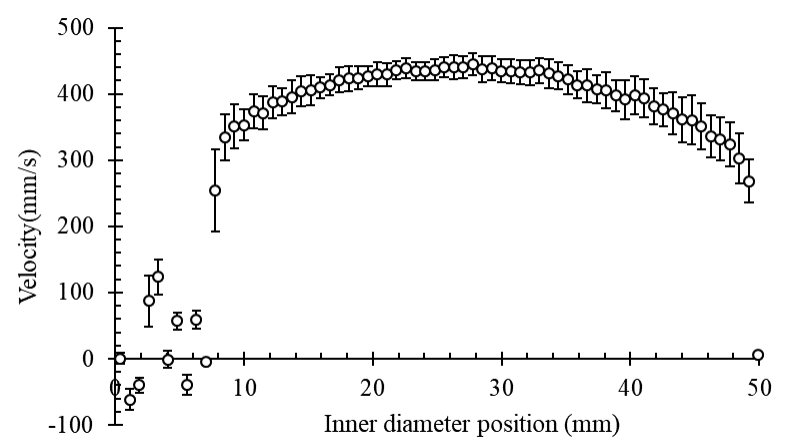

(a) Axial velocity $\left(V_{x}\right)$.

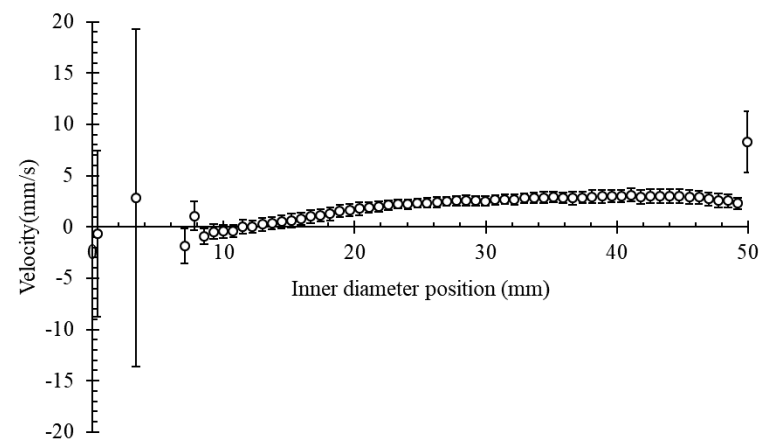

(b) Radial velocity $\left(V_{y}\right)$.

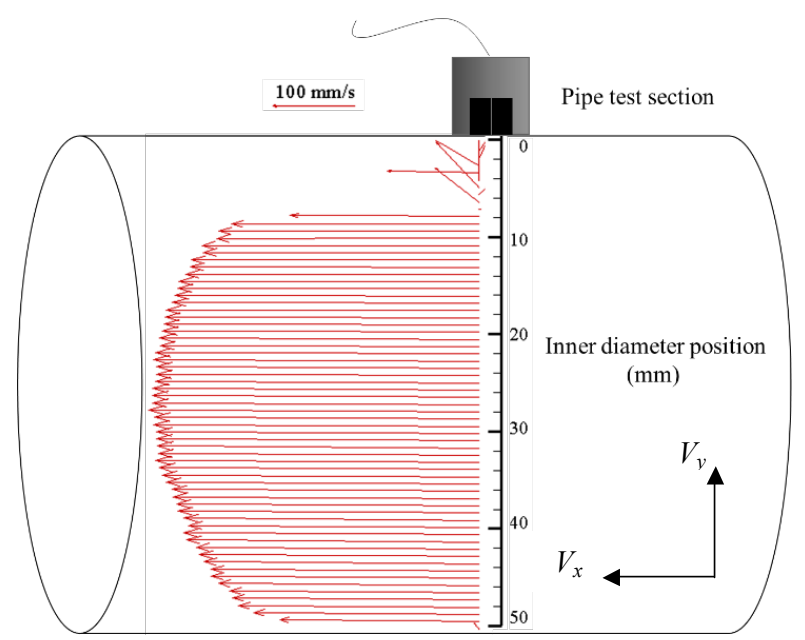

(c) Velocity vector.

Fig.12. Pipe flow measurement using two elements transducer.

From the result, we can see the two-dimensional velocity profile and the vector. The velocity in the axial direction $\left(V_{x}\right)$ has a high standard deviation compared to radial velocity $\left(V_{y}\right)$. The reason is the difference of the denominator from the equation of the axial velocity (equation (5)) and the radial velocity (equation (6)). The denominator in axial velocity is smaller compared to radial velocity. Therefore it will amplify the uncertainty or fluctuation of the profile and increase the standard deviation. In this measurement, the value of radial velocity is very small and has no significant effect on the vector reconstruction. We also can notice that in the region up to around $8 \mathrm{~mm}$, the profile is scattering. If we refer to the sound pressure, it is expected before to be not effective for two-dimensional measurement because the measurement volume does not focus in the center. Moreover from the simulation data, the near field also exist in that location and might reduce the measurement quality. To compare the profile, we use commercial single element transducer $(4 \mathrm{MHz}, 5 \mathrm{~mm})$ with an incident

Corresponding author: tanius.j.aa@m.titech.ac.jp 
angle $(\alpha)$ 10-degree to measure in the same flow condition and the result is presented as below.

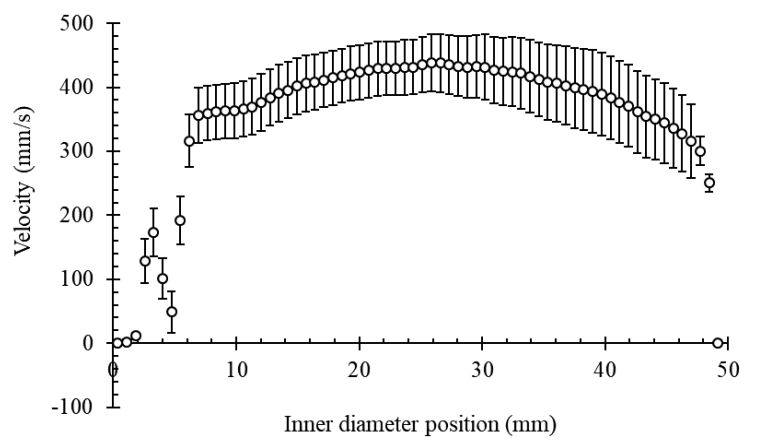

(a) Axial velocity $\left(V_{x}\right)$.

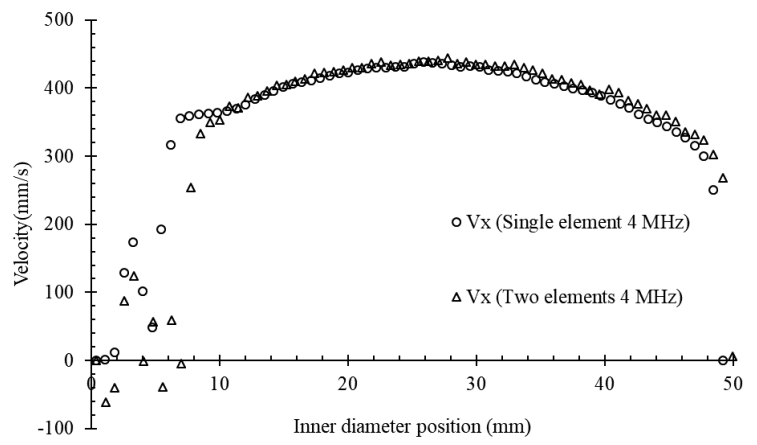

(c) Comparison with two elements transducer.

Fig.13. Pipe flow measurement using single element transducer.

From the profile comparison, it has a quite good agreement, except in the region up to $8 \mathrm{~mm}$. The flow rate can be calculated from integration of the profile (using equation (4)) and compared to the flow rate reading in Table 5 . The result is the two elements transducer measurement have larger error than single element transducer measurement. This fact might be caused by the profile scattering in two elements transducer profile at the region up to $8 \mathrm{~mm}$. Therefore the flow rate is lower than single element transducer. As for the standard deviation, the single element transducer has higher average value than the two element transducer. This might be caused by the noise effect of the shear wave. In the interface of solid, the wave will exist in two modes which are a shear wave and longitudinal wave (see Figure 14) entering the liquid. If there are two waves enter the liquid, one of them will become noise in the measurement, and usually, it is the shear wave with the lower transmission rate. This transmission rate can be observed from the transmission coefficient (the ratio of the transmission rate in working fluid to incident beam), and it depends on the incident angle (see Figure 15) [10]. In this case, the two elements transducer and single element transducer will have 0degree and 10-degree incident angle respectively. In 10degree incident angle (single element transducer), the noise transmission rate from shear wave will have higher value than 0-degree (two elements transducer). Therefore, in this single element transducer measurement, the noise effect cause the instantaneous velocity fluctuates.
Table 5. Flow measurement quality.

\begin{tabular}{|c|c|c|c|}
\hline Equipment & $\begin{array}{c}\text { Flow } \\
\text { rate } \\
(\mathbf{l} / \mathbf{m i n})\end{array}$ & $\begin{array}{c}\text { Error } \\
\mathbf{( \% )}\end{array}$ & $\begin{array}{c}\text { Average standard } \\
\text { deviation of } \\
\text { velocity } \\
\text { profile(mm/s) }\end{array}$ \\
\hline $\begin{array}{c}\text { Electromagnetic } \\
\text { flow meter } \\
\text { (reading) }\end{array}$ & 35 & - & - \\
\hline $\begin{array}{c}\text { Two elements } \\
\text { transducer }\end{array}$ & 33.26 & $4.98 \%$ & 22.20 \\
\hline $\begin{array}{c}\text { Single element } \\
\text { transducer }\end{array}$ & 33.90 & $3.14 \%$ & 45.74 \\
\hline
\end{tabular}

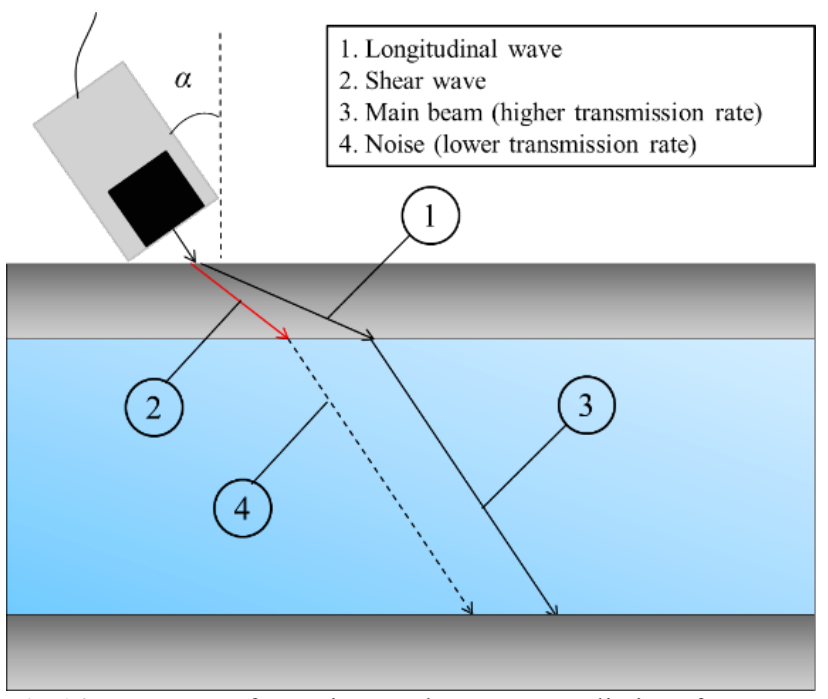

Fig.14. Wave transformation on the water-acrylic interface.

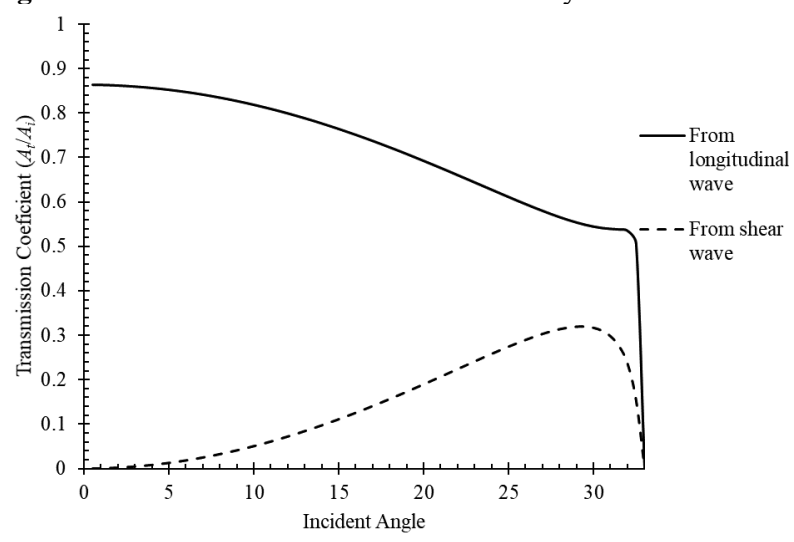

Fig.15. Theoretical transmission coefficient in the water depends on an incident angle with the acrylic-water interface.

\subsubsection{Pipe flow with swirl effect}

The measurement in this section is by adding swirl effect using a swirling generator in the distance of 3D before the flow reaches test section. The swirling effect is expected to increase the value of radial velocity and produce the not fully developed flow as we can see from the result presented in Figure 16. From the radial velocity profile, the value is higher compared to the pipe flow swirl effect. Therefore, we can see that the radial velocity has the significant effect to the flow direction in the velocity vector unlike in the straight pipe flow. For the comparison (see Figure 17), single element 
transducer is also used but with two incident angle (10degree and 45-degree) at this time, to see the effect of incident angle on not fully developed flow.

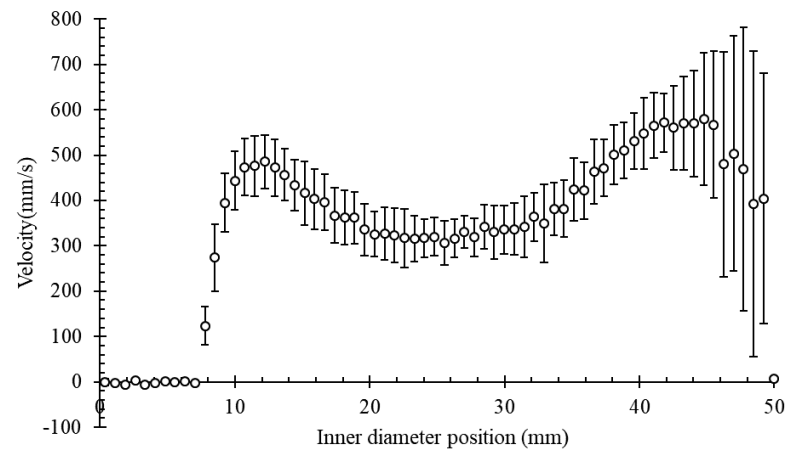

(a) Axial velocity $\left(V_{x}\right)$.

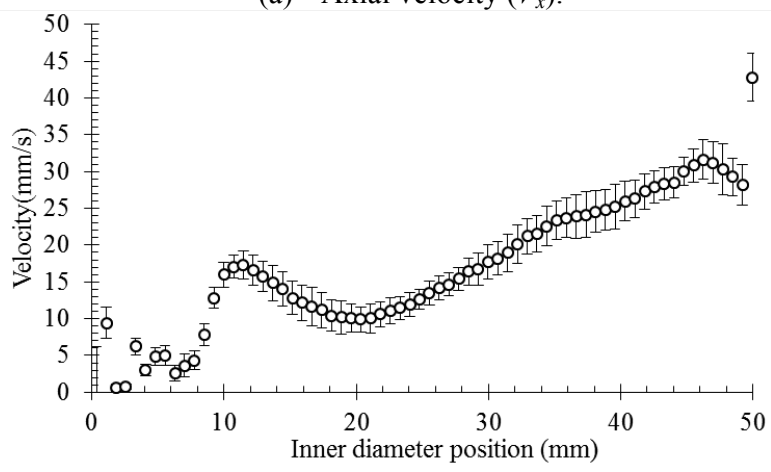

(b) Radial velocity $\left(V_{y}\right)$

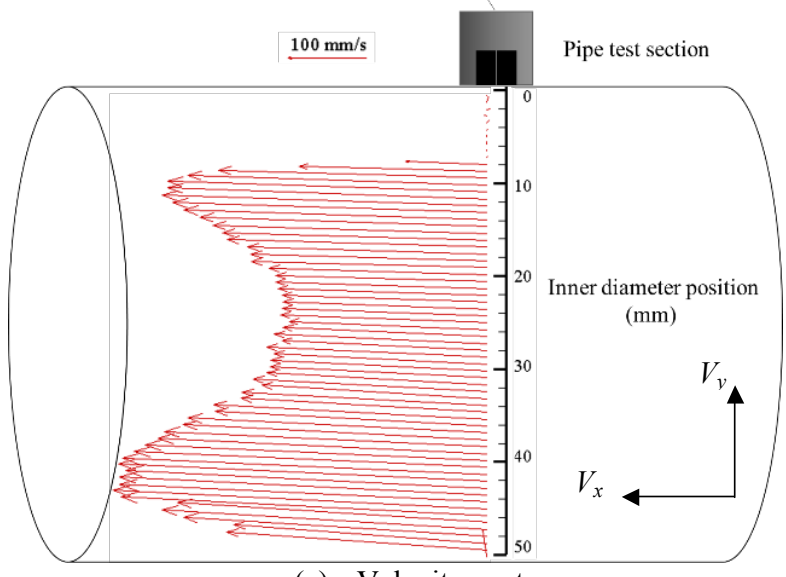

(c) Velocity vector.

Fig.16. Pipe flow measurement with swirl effect using two elements transducer.

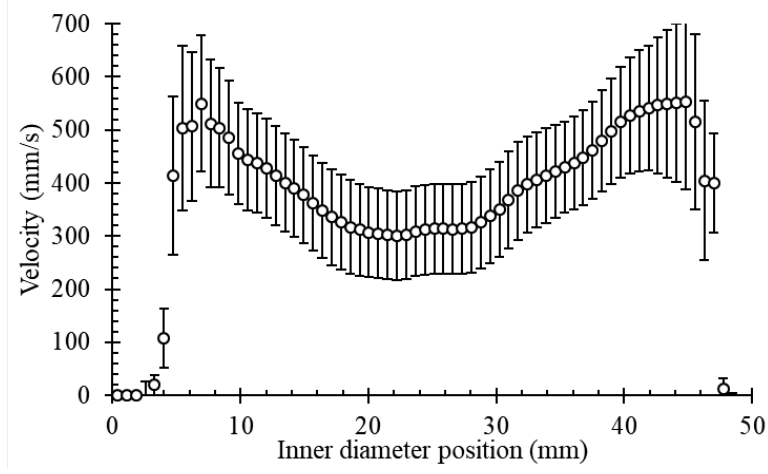

(a) Axial velocity $\left(V_{x}\right), 10$-degree incident angle.

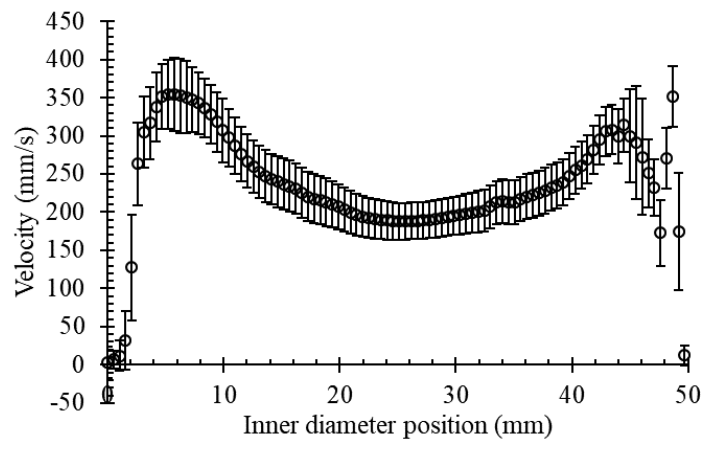

(b) Axial velocity $\left(V_{x}\right), 45$-degree incident angle.

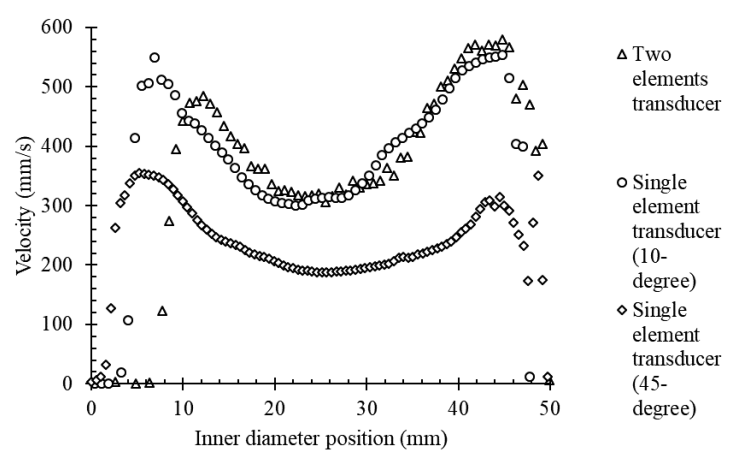

(c) Profile comparison.

Fig.17. Pipe flow measurement with swirl effect using single element transducer.

From the profile comparison, each profile is different to each other. This might be caused by the different incident angle measurement which results for each transducer measurement occurring in not a same position in the fluid while the flow is not fully developed. To evaluate the profile, the flow rate is calculated and compared to the reading flow rate.

Table 6. Flow measurement quality.

\begin{tabular}{|c|c|c|c|}
\hline Equipment & $\begin{array}{l}\text { Flow } \\
\text { rate } \\
(1 / \mathrm{min})\end{array}$ & $\begin{array}{c}\text { Error } \\
(\%)\end{array}$ & $\begin{array}{c}\text { Average standard } \\
\text { deviation of } \\
\text { velocity profile } \\
(\mathrm{mm} / \mathrm{s})\end{array}$ \\
\hline $\begin{array}{c}\text { Electro- } \\
\text { magnetic flow } \\
\text { meter } \\
\text { (reading) }\end{array}$ & 35 & - & - \\
\hline $\begin{array}{l}\text { Two elements } \\
\text { transducer }\end{array}$ & 38.65 & $10.43 \%$ & 71.57 \\
\hline $\begin{array}{c}\text { Single element } \\
\text { transducer, } 10 \\
\text { degree } \\
\text { incident angle }\end{array}$ & 40.05 & $14.42 \%$ & 90.02 \\
\hline $\begin{array}{c}\text { Single element } \\
\text { transducer, } 45 \\
\text { degree } \\
\text { incident angle }\end{array}$ & 28.91 & $17.41 \%$ & 34.16 \\
\hline
\end{tabular}

The overall result for this measurement has lower accuracy than previous measurement (without swirl effect). The reason is the flow under the swirl effect will have non-axisymmetric profile therefore velocity profile in only one measurement line does not fully represent the flow rate. For the profile comparison, the single 
element 45-degree incident angle have higher error compared to both single element 10-degree incident angle and two elements transducer. The reason is the measured area/position is very wide for not fully developed flow and the velocity profile in the measurement line and the flow profile will be different. Therefore, the calculation of flow rate will result in a higher error even with the small standard deviation. As for the two elements transducer, it has a better error than single element 10-degree incident angle and able to provide two-dimensional velocity profile information.

\subsubsection{Two elements transducer profile estimation}

One of the counter measure for the limitation in measurement range from two elements transducers is to estimate the profile in up to $8 \mathrm{~mm}$ region. The estimation can adopt statistic method and for this study we use Bspline interpolation with Gaussian fitting with the parameters of order 3 and knots 6 . The reason is this method can provide a fitting curve with high r-squared value $(0.995 \pm 0.003)$. Therefore, the estimation can be done while still keeping the original data (from $8 \mathrm{~mm}$ to $50 \mathrm{~mm}$ ) corresponding to the previous profile (Figure 12 and Figure 16). The interpolation is done by assuming the velocity in the first channel (nearest to the sensor and the wall) as zero. This method is applied for the twodimensional velocity measurement in pipe flow with and without swirl effect and the flow rate is calculated. The result is presented as below.

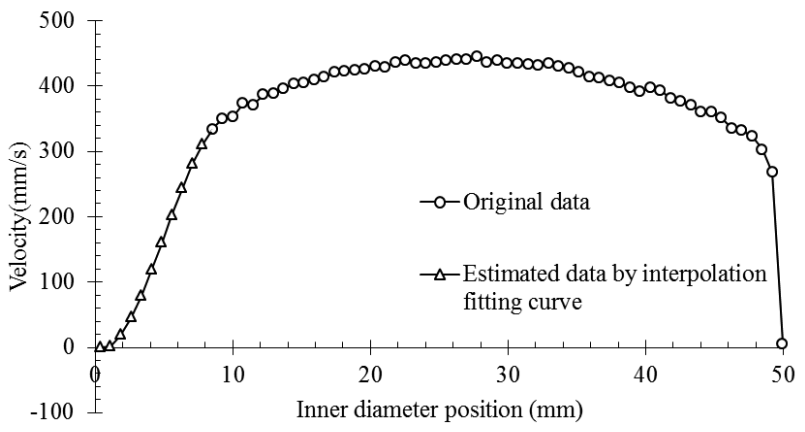

(a) Axial velocity $\left(V_{x}\right)$.

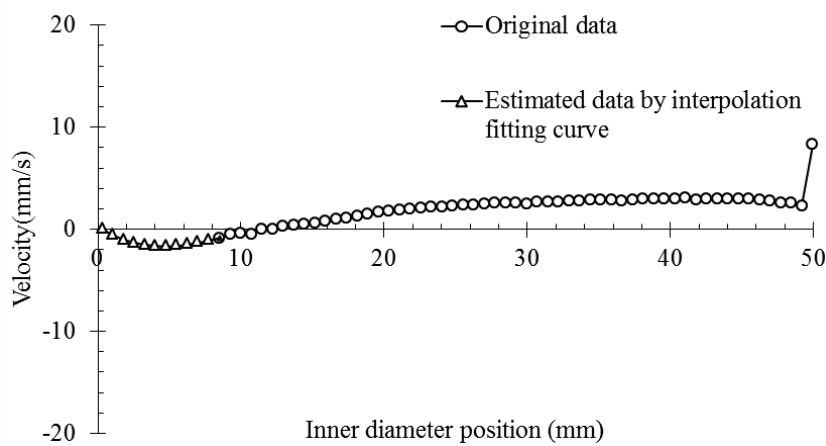

(b) Radial velocity $\left(V_{v}\right)$.

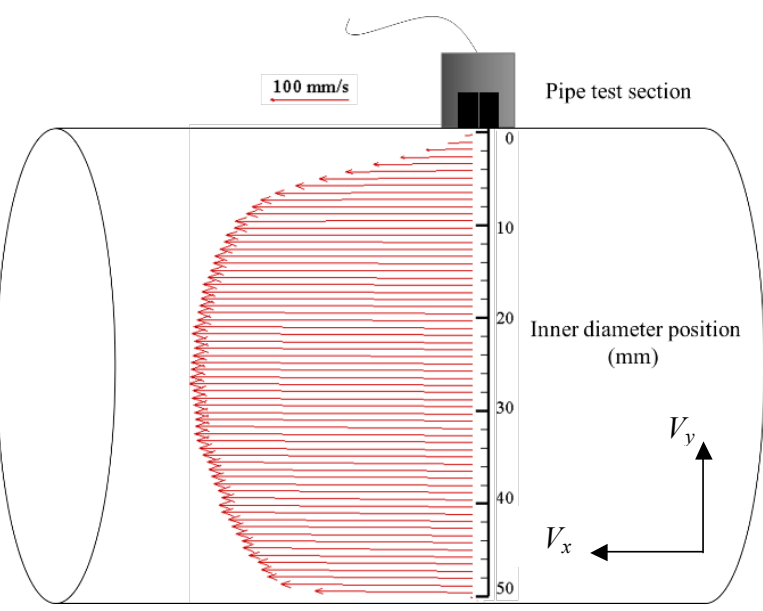

(c) Velocity vector.

Fig.18. Pipe flow measurement using two elements transducer with interpolation curve fitting (original data corresponding to profile in Figure 12)

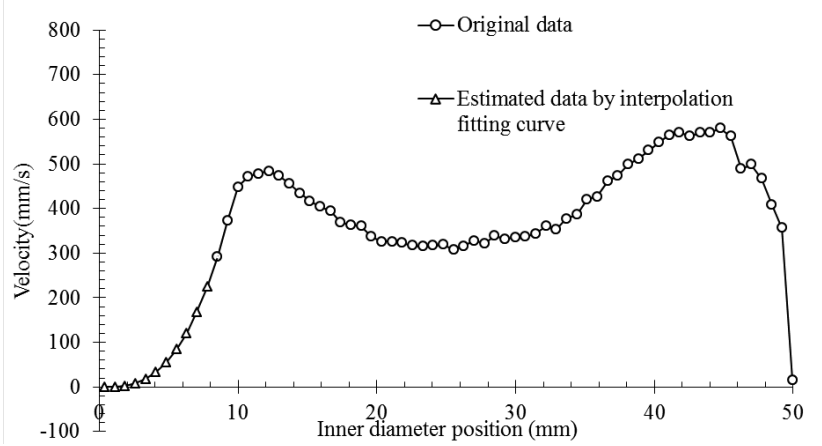

(a) Axial velocity $(\mathrm{Vx})$.

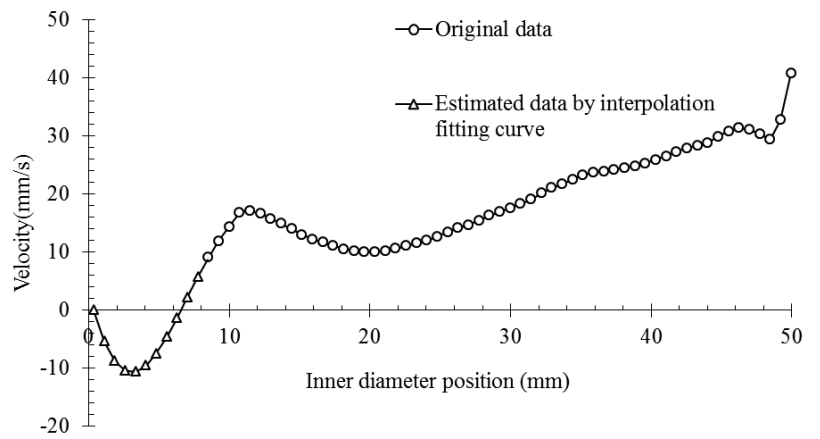

(b) Radial velocity (Vy).

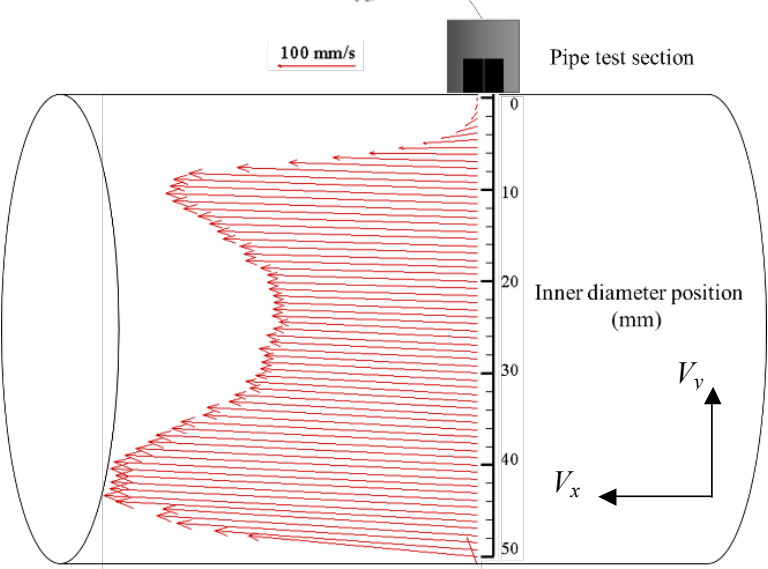

(c) Velocity vector.

Fig.19. Pipe flow measurement with swirl effect using two elements transducer with interpolation curve fitting. (original data corresponding to profile in Figure 16) 
Table 7. Flow rate calculation of the profile with interpolation curve fitting.

\begin{tabular}{|c|c|c|}
\hline Equipment & $\begin{array}{c}\text { Flow rate } \\
\text { (1/min) }\end{array}$ & $\begin{array}{c}\text { Error } \\
\text { (\%) }\end{array}$ \\
\hline $\begin{array}{c}\text { Electro-magnetic flow meter } \\
\text { (reading) }\end{array}$ & 35 & - \\
\hline $\begin{array}{c}\text { Two elements transducer } \\
\text { (without swirl effect) }\end{array}$ & 35.39 & $1.11 \%$ \\
\hline $\begin{array}{c}\text { Two elements transducer } \\
\text { (with swirl effect) }\end{array}$ & 39.52 & $12.92 \%$ \\
\hline
\end{tabular}

\section{Conclusion}

The two-dimensional velocity profile measurement system was developed by using only two elements transducer with both elements acting as a transceiver (transmitter and receiver). The system was designed with some targets and evaluated by sound pressure measurement and flow measurement. From the sound pressure measurement result, we confirm our design targets achieved with the effective measurement range start from $8 \mathrm{~mm}$. From the flow measurement result, we confirm the system capability in obtaining twodimensional velocity profile, and the flow rate can be calculated.

\section{References}

1. D.W. Baker, IEEE Trans. on Sonics and Ultrasonics. 17, 170-185 (1970)

2. B.L. Dunmire, K.W. Beach, K. Labs, M. Plett, D.E. Standness Jr, Ultrasound in Medicine and Biology, 26, 1213-1235 (2000)

3. P. Peronneau, W. Sandman, M. Xhaard, Ultrasound in Medicine, 1193-1208 (1977)

4. M. Scabia, M. Calzolai, L. Capineri, L. Masotti, A. Fort, Ultrasound Medicine and Biology, 26, 121131 (2000)

5. Y. Takeda. Nuclear Engineering and Design, 126, 277-284 (1991)

6. S.Wada, H.Kikura, M.Aritomi, M.Mori, Y.Takeda, Journal of Nuclear Science and Technology, 41, 339-346 (2004)

7. D.Huther and U.Lemmin, Measurement Science and Technology, 9, 10 (1998)

8. H. Obayashi, Y. Tasaka, S. Kon, Y. Takeda, Flow Measurement and Instrumentation, 19, 189-195 (2008)

9. C.Kasai, K.Namekawa, A.Koyano, R.Omoto, IEEE Transactions on sonics and ultrasonics, 32, 458-64 (1985)

10. S.Wada, K.Tezuka, W.Treenuson, N.suzuki, H.Kikura, Science and Technology of Nuclear Installations (2012) 